\title{
Flexible Interconnect of a Silicon Sensor at the Belle SVD
}

\author{
Koji Ueno, National Taiwan University, for the Belle SVD group, ueno@hep1.phys.ntu.edu.tw
}

\begin{abstract}
A flexible interconnect circuit(Flex) is employed for the upgraded Silicon Vertex Detector(SVD2) of the Belle experiment at the KEK b-factory. It brings signals of the Silicon Sensors to the outside of the SVD2 and makes them available for amplification at the Hybrid circuit. The required specification is rather difficult to meet. The conventional technology of a chemical etching process is stretched to the limit. We have a single-sided design as well as a double-sided design. We used a laser machine and repaired a bad spot such as a short circuit between two adjacent trace lines.
\end{abstract}

Index Terms - Flex, etching, spattering, wire-bonding, SVD, bfactory.

\section{INTRODUCTION}

$\mathbf{T}$ HE Belle experiment[1] at the KEK b-factory, Japan is upgrading the SVD in order to cope with higher luminosity and harder environment. The SVD2 has one more silicon layers for robust track reconstruction, a shorter ladder and a beampipe of a smaller radius of $1.5 \mathrm{~cm}$ for the better vertex measurement. It also has a longer ladder at a larger radius to cover a wider angle. Mechanical design and thermal consideration are more difficult. In order to have more flexibility in the design of the SVD upgrade, we employed the Flex in contrast to the Double Metal Structure(DMS) used in the first version of the SVD. The merits of the Flex over the DMS are: mechanical and thermal isolation of the silicon sensor from the hybrid circuit, ease of signal ganging and less capacitance in bringing out the silicon sensor signal.

The narrowest trace width of the Flex is $20 \mu \mathrm{m}$ and the largest surface area of the Flex is $28 \times 270 \mathrm{~mm}^{2}$. The conventional technology is stretched to the limit to make the Flex.

\section{REQUiRED SPECIFICATIONS}

The required specifications of the Flex are rather difficult to meet in a conventional technology. In the most difficult case, up to some 1500 trace lines must fit in a polyimid film of 28 X $270 \mathrm{~mm}^{2}$. The requirement is necessary to bring out the silicon signal along the longer ladder. The trace width is about $20 \mu \mathrm{m}$ and the pitch $40 \mu \mathrm{m}$. The wire-bonding pad is $40 \mathrm{X} 200$ $\mu m^{2}$ in size. See Fig. 1 for the actual pattern produced.

We have seven Flex types in total. We have three Flex types PS, PL, and PF to read out a $\phi$ coordinate. They range in size from $28 \times 32 \mathrm{~mm}^{2}$ to $35 \mathrm{X} 41 \mathrm{~mm}^{2}$ and their trace lines run parallel to the long dimension of Flex. We have four Flex types ZS, ZD, ZT, and ZF to read out a $\mathrm{z}$ coordinate. They

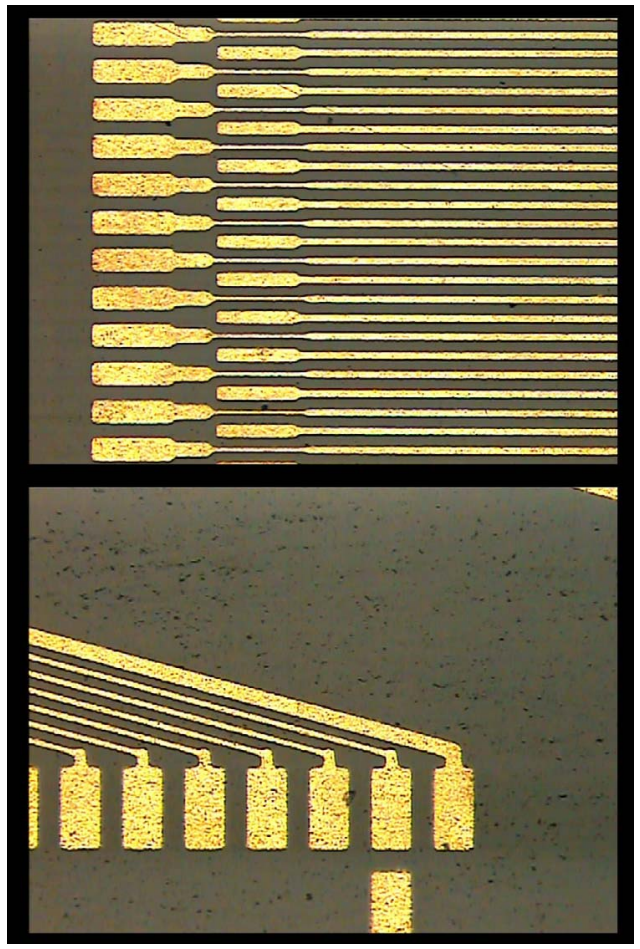

Fig. 1. A $\phi$ and $\mathrm{z}$ types and their bonding-pads. In the top figure of a $\phi$ type, the pad sizes are $40 \mathrm{X} 200 \mu \mathrm{m}^{2}$ and $80 \mathrm{X} 200 \mu \mathrm{m}^{2}$. The trace line width is 30 $\mu \mathrm{m}$ but about $10 \mu \mathrm{m}$ between the pads. In the bottom figure of a $\mathrm{Z}$ type, the pad size is $100 \times 200 \mu \mathrm{m}^{2}$ and the trace line width is $20 \mu \mathrm{m}$.

range in size from $28 \times 111 \mathrm{~mm}^{2}$ to $28 \mathrm{X} 270 \mathrm{~mm}^{2}$ and their trace lines have an angle of 20 deg. to the long dimension of Flex.

We decided the thickness of the trace by requiring the longest trace line has a resistance less than $50 \mathrm{ohms}$. The required trace thickness is $8 \mu \mathrm{m}$ in copper with the width of the trace of 20 $\mu \mathrm{m}$. The trace is narrower near the wire-bonding pads.

We require gold plating on the trace lines and wire-bonding pads for preventing corrosion of the traces and the pads and bonding wires on the pads. The base material is a polyimid. Its thickness is $50 \mu \mathrm{m}$.

\section{PATtern Formation}

We looked for a company, which can produce our Flex, in US, Europe, and Asia but did not find many. Finding a suitable company is most difficult but is important in the success of this project. We considered and evaluated different techniques 
for the pattern formation. We obtained engineering samples of Flex made by spattering, electrolytic, and chemical etching processes.

A spattering process produced a fine pattern of our Flex but had difficulty making the trace resistance small enough. A large size was also difficult to make at the time. An electrolytic process produced a fine pattern of our Flex but has a limitation in the design because the trace lines must be extended and connected to the electrodes of the electrolytic process. The extended trace lines can also cause a short circuit as they must be cut at the film edge.

We decided to employ the conventional chemical etching process. The technology must be stretched to the extreme limit because no company seems to be able to produce a fine pattern of our Flex routinely in a commercial base.

We have three designs of Flex: in the design A, all the patterns are on one side of the film; in the design $\mathrm{B}$, top and bottom sides of the film carry the pattern; in the design $\mathrm{C}$, two films carry the pattern and are laminated. The design B must bring a bottom trace to the top side via the through hole. The design $\mathrm{C}$ does not need the through hole to bring the bottom trace and has the over-sized bottom film to expose the pads. The component films is easier to make as the available space for traces and pads is doubled. We adopted the design A for SVD2 Flex[2]. We adopted the design C for the spares[3].

\section{QuALITY CONTROL}

Each trace line was inspected along its entire length with a microscope twice by a Flex company and by ourselves. When necessary, a laser microscope was used. It can show a 3-dimensional view of the trace line and can measure the trace thickness for example.

A sample of every batch was checked for bonding wires on the pads. We decided to do so after we had trouble bonding wires. The strength of bonded wires must be greater than 6 grams. The bad bonding turned out to be due to insufficient thickness of gold on the bonding pads. The gold thickness is usually $0.1 \mu \mathrm{m}$ but is increased to more than $0.3 \mu \mathrm{m}$ in the SVD2 Flex. A company for the spare Flex production proposes the gold thickness of more than $1 \mu \mathrm{m}$. Plating gold usually requires a nickel layer first. But some argues that it is not necessarily so and that forming an extra nickel layer causes damage to an already formed copper pattern. Because forming our pattern is extremely difficult, we decided not to have a nickel layer.

Because the quality of Flex was not satisfactory and, if we ask better quality, we can not meet our predetermined schedule, we decided to repair bad spots of Flex by a YAG laser machine. It can cut a short between two adjacent trace lines. The wave lengths used are $1064 \mathrm{~nm}$ and higher harmonics' and the laser spot size of a few $\mu \mathrm{m}$. We reduced the defect rate to $2 \%$. After the short is repaired, the resitance between the adjacent trace lines becomes about $1 \mathrm{M}$ ohms from about 0 ohms. See Fig. 2 for the bad spot before and after repairing.

The trace resistance was checked by hand and by a homemade automated machine on a sample basis. If measured

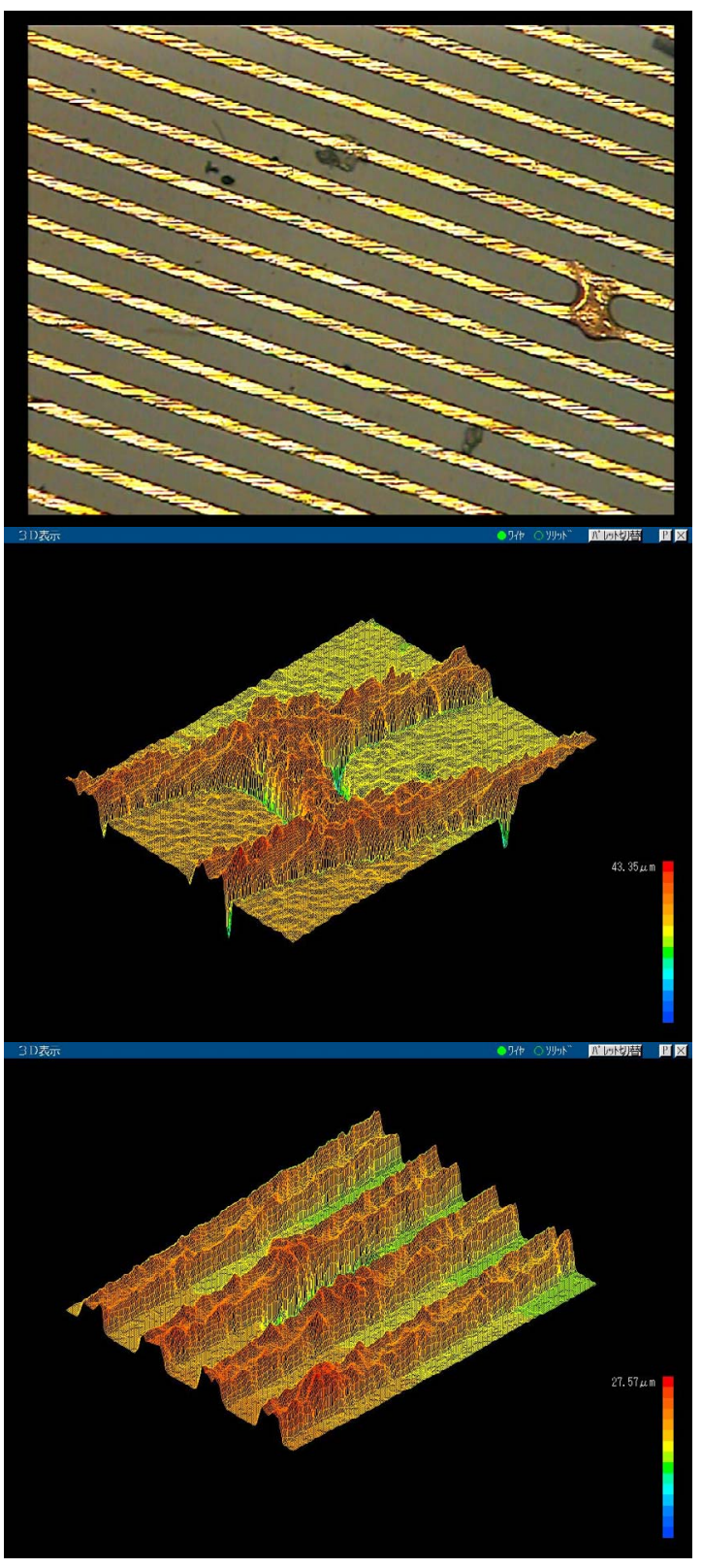

Fig. 2. A bad spot, a bad spot in 3-D view before repairing, and a bad spot in 3-D view after repairing.

manually, it takes five minutes per one trace. The machine can do the same in five seconds. It consists of a 3-dimensional moving table controled by a PC and a GPIB-interfaced R-C meter.

\section{CONCLUSION}

We have made Flex for the upgraded SVD in the Belle experiment at the KEK b-factory, Japan. The requirements of Flex are rather difficult to meet. We looked in available technologies and settled in chemical etching process for the pattern formation.

Flex has been assembled in the new SVD2 detector. SVD2 was installed in summer, 2003 and saw successfully the first 
track in Oct., 2003.

\section{ACKNOWLEDGMENT}

The author would like to thank Wei-Chun Lin and I-Dong Chewo for laying out the Flex design.

\section{REFERENCES}

[1] Nucl. Instr. and Meth. A479(2002) 117.

[2] Keycom Co, Tokyo, Japan.

[3] Dyconex Ltd, Basserdorf, Switzerland. 